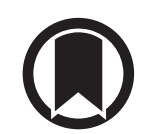

CrossMark

\title{
The Tuberculosis Network European Trials group (TBnet) ERS Clinical Research Collaboration: addressing drug-resistant tuberculosis through European cooperation
}

\author{
Frank van Leth (10 ${ }^{1,2}$, Folke Brinkmann ${ }^{3}$, Daniela M. Cirillo ${ }^{4}$, Keertan Dheda ${ }^{5,6}$, \\ Raquel Duarte ${ }^{7,8}$, Lorenzo Guglielmetti $\mathbb{1}^{9,10}$, Liga Kuksa ${ }^{11}$, \\ Christoph Lange ${ }^{12,13}$, Carole Mitnick ${ }^{14}$, Alena Skrahina ${ }^{15}$, Khalequ Zaman ${ }^{16}$ and \\ Graham Bothamley ${ }^{17,18,19}$
}

\begin{abstract}
Affiliations: ${ }^{1}$ Amsterdam Institute for Global Health and Development, Amsterdam, The Netherlands. ${ }^{2}$ Amsterdam University Medical Centers, Location Meibergdreef, Amsterdam Public Health Research Institute, University of Amsterdam, Amsterdam, The Netherlands. ${ }^{3}$ Universitaetskinderklinik fuer Kinder- und Jugendmedizin, Ruhr University, Bochum, Germany. ${ }^{4} E$ merging Bacterial Pathogens, IRCCS San Raffaele Scientific Institute, Milan, Italy. ${ }^{5}$ Centre for Lung Infection and Immunity, UCT Lung Institute and Dept of Medicine, University of Cape Town, Cape Town, South Africa. ${ }^{6}$ University College London, London, UK. ${ }^{7}$ Pneumology Dept, Centro Hospitalar Vila Nova de Gaia, Vila Nova de Gaia, Portugal. ${ }^{8}$ ISPUP-EP unit, Faculty of Medicine, University of Porto, Porto, Portugal. ${ }^{9}$ APHP, Centre National de Référence des Mycobactéries et de la Résistance des Mycobactéries aux Antituberculeux (CNR-MyRMA), Bactériologie-Hygiène, Hôpitaux Universitaires Pitié Salpêtrière-Charles Foix, Paris, France. ${ }^{10}$ Sorbonne Université, Université Pierre et Marie Curie 06, Unité 1135, Team E13 (Bactériologie), CR7 INSERM, Centre d'Immunologie et des Maladies Infectieuses, Paris, France. ${ }^{11}$ Riga East University Hospital, and WHO Collaborating Centre, Riga, Latvia. ${ }^{12}$ German Center for Infection Research Tuberculosis Unit, Research Center Borstel, Borstel, Germany. ${ }^{13}$ Clinical Infectious Diseases, Research Center Borstel, Borstel, Germany. ${ }^{14}$ Dept of Global Health and Social Medicine, Harvard Medical School, Boston, MA, USA. ${ }^{15}$ The Republican Research and Practical Centre for Pulmonology and Tuberculosis, Minsk, Belarus. ${ }^{16}$ ICDDRB Infectious Disease Division, Dhaka, Bangladesh. ${ }^{17}$ Homerton University Hospital, London, UK. ${ }^{18}$ Blizard Institute, Barts and The Royal London School of Medicine and Dentistry, Queen Mary University of London, London, UK. ${ }^{19}$ London School of Hygiene and Tropical Medicine, London, UK.
\end{abstract}

Correspondence: Frank van Leth, Amsterdam Institute for Global Health and Development, Paasheuvelweg 25, Amsterdam, 1105 BP, The Netherlands. E-mail: f.vanlethaaighd.org

@ERSpublications

The Tuberculosis Network European Trials group (TBnet) continues the successful ERS Clinical Research Collaboration on drug-resistant tuberculosis http://ow.ly/gGH330mHrDa

Cite this article as: van Leth F, Brinkmann F, Cirillo DM, et al. The Tuberculosis Network European Trials group (TBnet) ERS Clinical Research Collaboration: addressing drug-resistant tuberculosis through European cooperation. Eur Respir J 2019; 53: 1802089 [https://doi.org/10.1183/13993003.02089-2018].

While the incidence of tuberculosis (TB) has fallen steadily within the European Union, the region has by far the highest estimated incidence of multidrug-resistant (MDR)-TB among TB patients [1, 2], which hampers the goal of the World Health Organization (WHO) of TB elimination in the region [3]. MDR-TB has the potential to be the most important infectious threat to European health over the coming decades, unless effective treatment can be found and relapses prevented. 
The European Respiratory Society (ERS) established the mechanism of Clinical Research Collaborations (CRCs) to facilitate the collaboration between healthcare professionals, scientists, regulators and patients. The mechanism promotes cross-disciplinary research and builds a platform for clinical research in the field of respiratory medicine [4]. The Tuberculosis Network European Trials group (TBnet) was the first to develop the idea of an ERS CRC in 2009 and has concentrated on MDR-TB since 2012. This has provided the network with invaluable administrative support and embedded the work of TBnet clearly in the work of ERS through Assembly 10 (Respiratory Infections), and particularly with group 10.02 (TB) (figure 1).

The ERS CRC in MDR-TB by TBnet 1) raises awareness of the importance of MDR-TB in Europe, 2) provides a platform for multidisciplinary research in MDR-TB, 3) collects data from operational settings to improve diagnosis and treatment of MDR-TB, 4) addresses health inequalities in access to diagnostic tools and treatment, and 5) provides essential capacity building for early career clinicians and researchers.

\section{TBnet}

TBnet (www.tbnet.eu) is a pan-European network consisting of over 600 healthcare professionals and scientists, whose aim is to promote quality of care for TB patients through research and education [5]. It has two daughter organisations, ptbnet (for paediatric TB) and NTM-NET (for nontuberculous mycobacteria). So far, the collaborative research of TBnet has resulted in more than 60 peer-reviewed articles in leading medical journals.

Multicentre clinical studies are conducted by collaborations among the network's members. This is a departure from the work of conventional research groups where activities are steered by a small group of participants. As such, the network utilises country-specific expertise to its best advantage and embeds its activities in the operational characteristics of its members.

The educational flagship of TBnet is its annual Academy, a 3-day masterclass for early career clinicians and scientists. Selected participants are mentored before the start of the Academy by the TBnet faculty to prepare state-of-the-art lectures on key topics for the other participants during the Academy. This strategy stimulates development of international perspectives and kick-starts the creation of a participant's professional network. The Academy has taken place in Central/Eastern Europe (Moldova, Ukraine, Armenia, Russia) to limit barriers for participation.

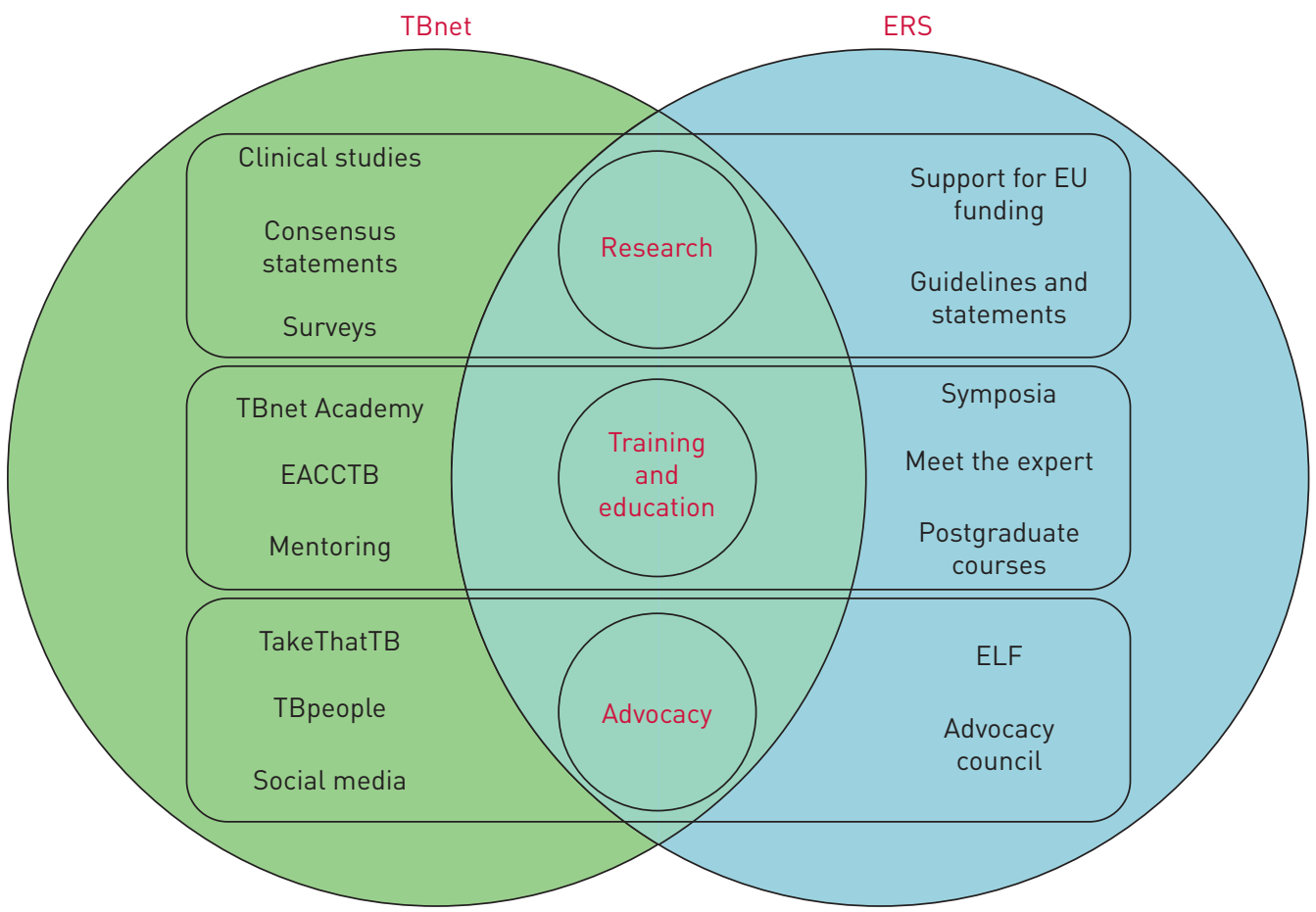

FIGURE 1 Intersections between the Tuberculosis Network European Trials group (TBnet) and European Respiratory Society (ERS) Assembly 10 in the ERS Clinical Research Collaboration. EU: European Union; EACCTB: European Advanced Course in Clinical Tuberculosis; ELF: European Lung Foundation. 
The European Advanced Course in Clinical Tuberculosis (EACCTB) is a 3-day course with a strong focus on clinical management, geared towards young medical professionals. It has been organised in collaboration with international partners (KNCV Tuberculosis Foundation (from the Netherlands), Filha (the Finnish Lung Health Association), the Karolinska Institute (Stockholm, Sweden), and the Hospital and University of Tartu, Estonia).

Membership of TBnet is free of charge. The network provides a platform where every member can propose new studies or participate in ongoing studies. TBnet is supporting data sharing as part of an "open science" environment. Requests for access to data collected through TBnet studies are reviewed by TBnet's Steering Committee to ensure the requested data can contribute to the proposed research question. Several TBnet studies have been included in individual patient data meta-analyses, thereby strengthening worldwide research collaborations.

All TBnet activities are reported and discussed at the network's annual meeting, which is scheduled for the day before the start of the ERS International Congress, to underline the close relationship between the two organisations.

\section{TBnet's achievements}

TBnet has made significant contributions to the management of $\mathrm{TB}$, regarding the use of interferon- $\gamma$ release assays, molecular testing for drug resistance, evaluating short-course chemotherapy for MDR-TB in Europe, and recommendations for outcome definitions [6].

Within the 2015-2018 ERS CRC, TBnet continued to expand its network of health facilities specialised in care for patients with MDR-TB. Of these, 23 facilities from 16 different European countries provided in-depth information on the magnitude and clinical management of drug-resistant TB in Europe, by implementing a European Union 7th Framework Programme-funded work package in the TB PAN-NET project (figure 2) [7].

The work showed the paucity of drug-sensitivity testing for second-line drugs other than fluoroquinolones and injectables $[8,9]$, mainly due to the absence of reliable tools for patients with MDR-TB. When available, drug-sensitivity testing revealed a high prevalence of additional drug resistance for ethambutol,

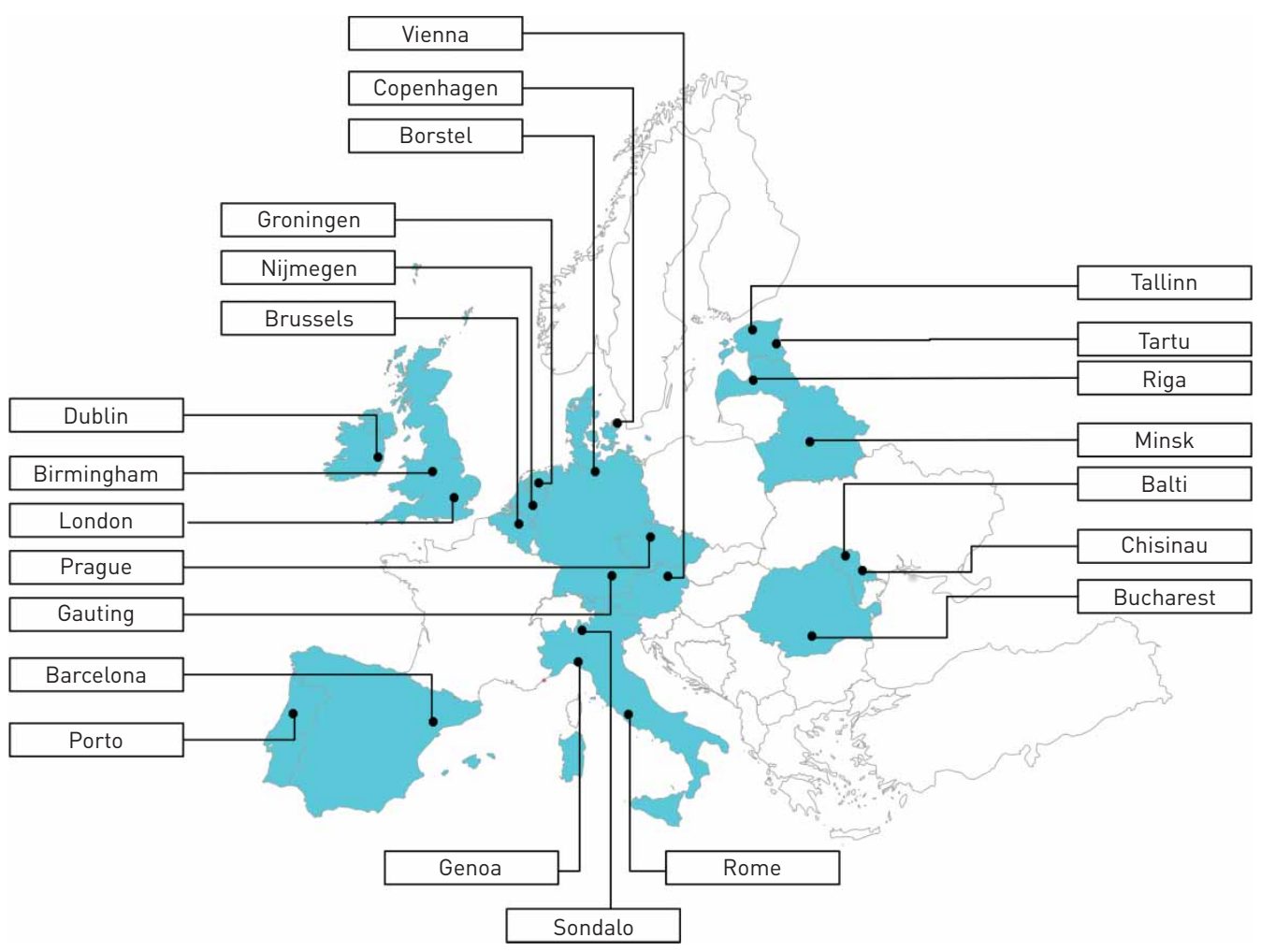

FIGURE 2 The Tuberculosis Network European Trials group (TBnet) health facilities collaborating in the clinical cohort studies. 
pyrazinamide and protionamide/ethionamide, as well as moxifloxacin and kanamycin, especially in high-TB-incidence countries within Europe $[8,9]$. All these drugs are key components of the short regimen for MDR-TB, resulting in limited scope of this regimen, with on average only $8 \%$ of patients with MDR-TB in Europe being eligible [10].

TBnet identified marked inequalities between low-, middle- and high-TB-incidence countries in Europe in access to initial treatment regimens for MDR-TB. Over $90 \%$ of MDR-TB patients were started on a personalised regimen in low-TB-incidence countries, compared to just over $15 \%$ in high-TB-incidence countries [11]. In these high-incidence countries, the high resistance to second-line drugs at the start of treatment and the higher frequency of acquired drug-resistance during therapy would seem to contradict the higher frequency of treatment success observed in these settings, compared to patients from low-TB-incidence countries, when applying WHO-defined treatment outcomes [11].

The WHO cure definition's reliance on at least three consecutive negative cultures in the continuation phase of MDR treatment is problematic. Only 13\% of MDR-TB patients in low-incidence countries and $52 \%$ in high-incidence countries had three samples collected in that phase of treatment [12]. These figures are even lower for patients with drug-sensitive TB [13]. As a reaction to this finding, TBnet proposed revised TB treatment outcome definitions with three key concepts: 1) cure is defined independently of the number of sputum cultures in the continuation phase, 2) failure is defined independently of the change of treatment and duration of treatment, to reflect settings where drugs cannot be changed due to unavailability, and 3) incorporation of an observation period after treatment cessation to identify possible relapse $[12,14]$. These changes make it possible to have identical classifications for all settings of $\mathrm{TB}$ treatment, including drug-sensitive TB and the paediatric population [15].

TBnet is able to elicit opinions and perform situation analyses through web-based surveys. Using focal points in participating countries, the network estimated the incidence of QT-prolongation and cardiotoxicity of currently used drugs [16], identified large inequalities in availability of TB drugs and in the price for a complete treatment course throughout Europe [17], mapped strategies in bacille CalmetteGuérin vaccination and their changes due to shortage of the vaccine [18, 19], identified key strategies to manage child MDR-TB contacts [20], and identified gaps in infection control strategies in the management of patients with drug-resistant TB in high-incidence countries [21].

The rapidly changing landscape in diagnostic tests and treatment demands guidance for clinical management long before formal scientific systematic reviews can be performed or are available. TBnet regularly combines expertise available in the network with key stakeholders within and outside the network, to arrive at well-founded consensus statements. The topics have been diverse and have included latent TB infection [22], contact investigation strategies [23] and diagnosis and treatment guidelines [24-27]. TBnet's publication authorship acknowledges the central role that frontline staff, rather than established investigators, make to healthcare research.

TBnet has established close collaborations with patient and advocacy groups, especially TakeThatTB and TBpeople. This ensures an increasing patient-centred view on TB-control activities.

\section{Future plans}

The aims for TBnet's 2018-2021 ERS CRC are ambitious. First, clinicians need to be able to manage MDR-TB with confidence. There is a need to update the consensus statements on molecular drug-sensitivity testing for Mycobacterium tuberculosis [24] and the management of patients with MDR-TB [25]. It is anticipated that both consensus statements will provide a welcome basis for clinicians in their everyday work with patients with drug-resistant $\mathrm{TB}$, thereby providing guidance on the use of newly available drugs and diagnostic tools, and improving the quality of care and treatment outcomes.

Secondly, there is a need for identifying best practices for inclusive care for TB among individuals new to Europe. A new TBnet consensus statement will cover a wide range of topics, including latent infections, screening, diagnosis, management, drug resistance, access to care, and legal frameworks, informing clinicians and policy makers alike on the critical task of providing inclusive care for TB patients.

Thirdly, accurate and meaningful data from operational settings need to be gathered. The clinical network is being strengthened and expanded for new data collection, which will capture the effects of introducing new treatment guidelines and access to new diagnostics. Special attention is given to the relationship between health inequalities and treatment outcomes. The "lessons learned" from the previous cohort will enable planning for a more robust use of routine data to emulate clinical trials. This is the most efficient approach when large-scale randomised clinical trials to estimate treatment efficacies of regimens are difficult or impossible $[28,29]$. The cohort will provide crucial information on the safety of new TB drugs 
when used in routine care, and will provide a population for the validation of the proposed treatment outcome definitions. The strength of TBnet's cohort approach has been clearly recognised [30].

Fourthly, TBnet will align its advocacy activities more with the European Lung Foundation.

All these tasks require junior researchers, whose scientific development can be encouraged through the TBnet Academy and by sharing of up-to-date knowledge with established healthcare professionals through the EACCTB and ERS International Congress.

\section{Conclusion}

The ERS CRC mechanism provides TBnet with invaluable support, and embeds the network firmly in the wider activities of the ERS, by facilitating cross-disciplinary cooperation and liaison with other CRCs. The mechanism fosters capacity building of early career physicians and scientists, as well as cutting-edge research in the field of respiratory diseases in order to improve clinical care and outcomes of patients with MDR-TB.

Conflict of interest: F. van Leth has nothing to disclose. F. Brinkmann has nothing to disclose. D.M. Cirillo has nothing to disclose. K. Dheda has nothing to disclose. R. Duarte has nothing to disclose. L. Guglielmetti has nothing to disclose. L. Kuksa has nothing to disclose. C. Lange reports personal fees (sponsorship for independent lectures at sponsored symposia) from Chiesi, Gilead, Abbvie, MSD, Becton Dickinson, Janssen, Lucane, Novartis and Thermofisher, outside the submitted work. C. Mitnick has nothing to disclose. A. Skrahina has nothing to disclose. K. Zaman has nothing to disclose. G. Bothamley reports that the TBnet ERS CRC receives a grant from the ERS.

\section{References}

1 World Health Organization. Global Tuberculosis Report 2018. WHO/CDS/TB/2018.20. Geneva, World Health Organization, 2018. Available from: www.who.int/tb/publications/global_report/en/

2 European Centre for Disease Prevention and Control, WHO Regional Office for Europe. Tuberculosis Surveillance and Monitoring in Europe 2018. 2016 data. Stockholm, European Centre for Disease Prevention and Control, 2018. Available from: https://ecdc.europa.eu/en/publications-data/tuberculosis-surveillance-and-monitoring-europe-2018

3 World Health Organization. The End TB Strategy. www.who.int/tb/strategy/end-tb/en/ Date last accessed: October 15, 2018.

4 Brightling C, Genton C, Bill W, et al. ERS Clinical Research Collaborations: underpinning research excellence. Eur Respir J 2018; 52: 1801534.

5 Giehl C, Lange C, Duarte R, et al. TBNET - Collaborative research on tuberculosis in Europe. Eur J Microbiol Immunol 2012; 2: 264-274.

6 Bothamley G. The Tuberculosis Network European Trials Group (TBNET): new directions in the management of tuberculosis. Breathe 2017; 13: e65-e71.

7 European Commission Community Research and Development Information Service (CORDIS). Final Report Summary - TB PAN-NET (Pan-European network for the study and clinical management of drug resistant tuberculosis). https://cordis.europa.eu/result/rcn/159240_en.html Date last accessed: October 13, 2018. Date last updated: April 8, 2015.

8 Günther G, van Leth F, Alexandru S, et al. Multidrug-resistant tuberculosis in Europe, 2010-2011. Emerg Infect Dis 2015; 21: 409-416.

9 Günther G, van Leth F, Altet N, et al. Beyond multidrug-resistant tuberculosis in Europe: a TBNET study. Int J Tuberc Lung Dis 2015; 19: 1524-1527.

10 Lange C, Duarte R, Fréchet-Jachym M, et al. Limited benefit of the new shorter multidrug-resistant tuberculosis regimen in Europe. Am J Respir Crit Care Med 2016; 194: 1029-1031.

11 Günther $\mathrm{G}$, van Leth $\mathrm{F}$, Alexandru $\mathrm{S}$, et al. Clinical management of multidrug-resistant tuberculosis in 16 European countries. Am J Respir Crit Care Med 2018; 198: 379-386.

12 Günther G, Lange C, Alexandru S, et al. Treatment outcomes in multidrug-resistant tuberculosis. N Engl J Med 2016; 375: 1103-1105.

13 Dedicoat MJ, Günther G, Crudu V, et al. Tuberculosis treatment outcomes in Europe: based on treatment completion, not cure. Am J Respir Crit Care Med 2017; 196: 1222-1224.

14 Lange $\mathrm{C}$, van Leth F, Mitnick CD, et al. Time to revise WHO-recommended definitions of MDR-TB treatment outcomes. Lancet Respir Med 2018; 6: 246-248.

15 Heyckendorf J, van Leth F, Kalsdorf B, et al. Relapse-free cure from multidrug-resistant tuberculosis in Germany. Eur Respir J 2018; 51: 1702122.

16 Guglielmetti L, Tiberi S, Burman M, et al. QT prolongation and cardiac toxicity of new tuberculosis drugs in Europe: a Tuberculosis Network European Trialsgroup (TBnet) study. Eur Respir J 2018; 52: 1800537.

17 Günther G, Gomez GB, Lange C, et al. Availability, price and affordability of anti-tuberculosis drugs in Europe: a TBNET survey. Eur Respir J 2015; 45: 1081-1088.

18 Dierig A, Tebruegge M, Krivec U, et al. Current status of Bacille Calmette Guérin (BCG) immunisation in Europe - a ptbnet survey and review of current guidelines. Vaccine 2015; 33: 4994-4999.

19 Kontturi A, Santiago B, Tebruegge M, et al. The impact of Bacille Calmette-Guérin shortage on immunisation practice and policies in Europe - a Paediatric Tuberculosis Network European Trials Group (ptbnet) survey. Tuberculosis 2016; 101: 125-129.

20 Turkova A, Tebruegge M, Brinkmann F, et al. Management of child MDR-TB contacts across countries in the WHO European Region: a survey of current practice. Int J Tuberc Lung Dis 2017; 21: 774-777. 
21 Bothamley GH, Lange C. Infection control, genetic assessment of drug resistance and drug susceptibility testing in the current management of multidrug/extensively-resistant tuberculosis (M/XDR-TB) in Europe: a tuberculosis network European Trialsgroup (TBNET) study. Respir Med 2017; 132: 68-75.

22 Mack U, Migliori GB, Sester M, et al. LTBI: latent tuberculosis infection or lasting immune responses to M. tuberculosis? A TBNET consensus statement. Eur Respir J 2009; 33: 956-973.

23 Erkens CG, Kamphorst M, Abubakar I, et al. Tuberculosis contact investigation in low prevalence countries: a European consensus. Eur Respir J 2010; 36: 925-949.

24 Domínguez J, Boettger EC, Cirillo D, et al. Clinical implications of molecular drug resistance testing for Mycobacterium tuberculosis: a TBNET/RESIST-TB consensus statement. Int J Tuberc Lung Dis 2016; 20: 24-42.

25 Lange C, Abubakar I, Alffenaar JW, et al. Management of patients with multidrug-resistant/extensively drugresistant tuberculosis in Europe: a TBNET consensus statement. Eur Respir J 2014; 44: 23-63.

26 Solovic I, Sester M, Gomez-Reino JJ, et al. The risk of tuberculosis related to tumour necrosis factor antagonist therapies: a TBNET consensus statement. Eur Respir J 2010; 36: 1185-1206.

27 Bumbacea D, Arend SM, Eyuboglu F, et al. The risk of tuberculosis in transplant candidates and recipients: a TBNET consensus statement. Eur Respir J 2012; 40: 990-1013.

28 Hernán MA, Alonso A, Logan R, et al. Observational studies analyzed like randomized experiments: an application to postmenopausal hormone therapy and coronary heart disease. Epidemiology 2008; 19: 766-779.

29 Hernán MA, Robins JM. Estimating causal effects from epidemiological data. J Epidemiol Community Health 2006; 60: 578-586.

30 Monedero-Recuero I. Drug-resistant tuberculosis in Europe. What are we waiting for? Am J Respir Crit Care Med 2018; 198: 302-304. 